\title{
On the perpendicular diffusion of solar energetic particles
}

\author{
R D Strauss*† \\ Center for Space Research, North-West University Potchefstroom, South Africa \\ E-mail: dutoit.strauss@nwu.ac.za
}

\section{H Fichtner}

Institut für Theoretische Physik IV, Ruhr Universität, Bochum, Germany

E-mail: hfetp4.rub.de

\begin{abstract}
The multitude of recent multi-point spacecraft observations of solar energetic particle (SEP) events have made it possible to study the longitudinal distribution of SEPs in great detail. SEPs, even those accelerated during impulsive events, show a much wider than expected longitudinal extent, bringing into question the processes responsible for their transport perpendicular to the local magnetic field. In this paper we examine some aspects of perpendicular transport by including perpendicular diffusion into a numerical SEP transport model that simulates the propagation of impulsively accelerated SEP electrons. We find that: (i) SEP intensities are generally asymmetric in longitude, being enhanced towards the west of optimal magnetic connection to the acceleration region. (ii) The maximum SEP intensity may also be shifted (parameter dependently) away from the longitude of best magnetic connectivity at $1 \mathrm{AU}$. We also calculate the maximum intensity, the time of maximum intensity, the onset time and the maximum anisotropy as a function of longitude at Earth's orbit and compare the results, in a qualitative fashion, to recent spacecraft observations.
\end{abstract}

The 34th International Cosmic Ray Conference,

30 July- 6 August, 2015

The Hague, The Netherlands

\footnotetext{
* Speaker.

${ }^{\dagger}$ Currently on sabbatical leave at: The Center for Space Plasma and Aeronomic Research (CSPAR), University of Alabama in Huntsville, USA
} 

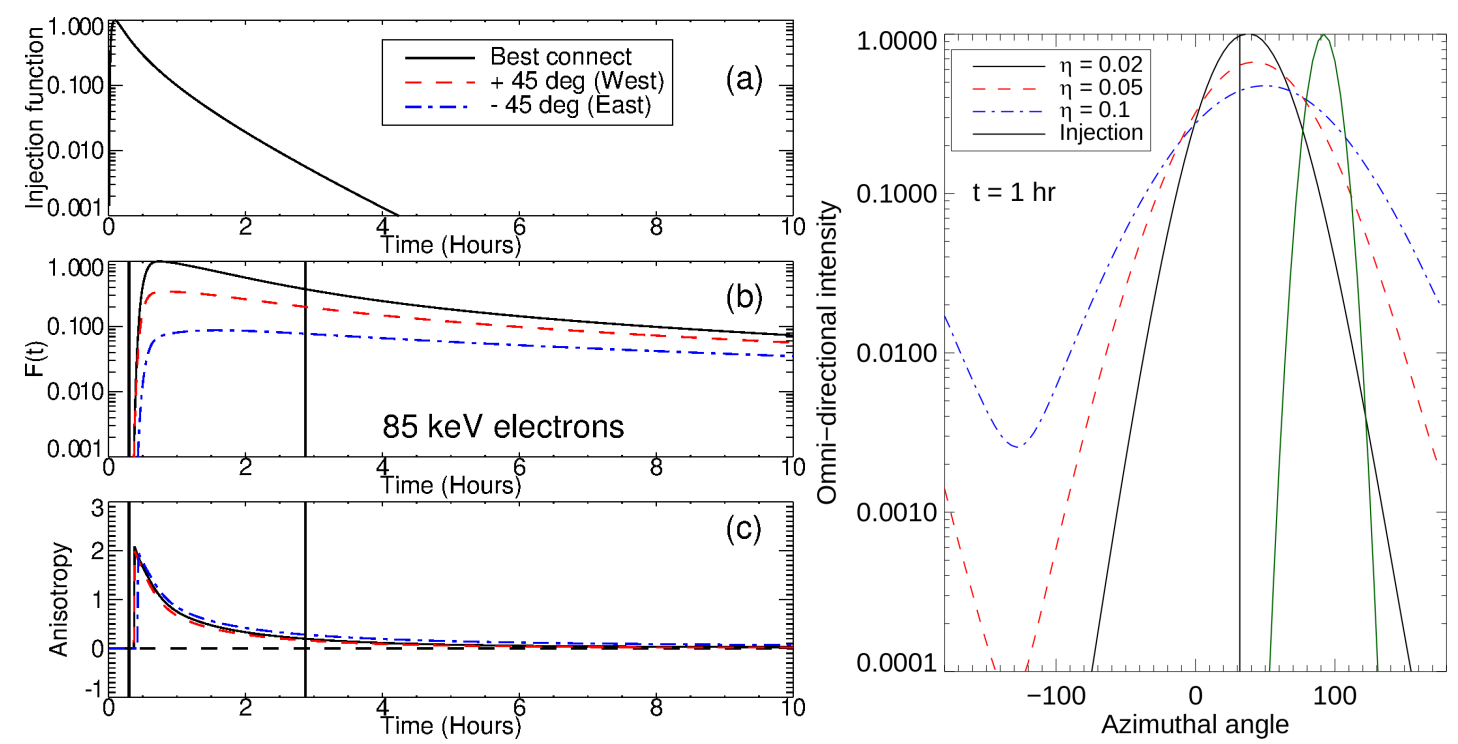

Figure 1: Left: The top panel shows the assumed injection function, while panels (b) and (c) show the resulting omni-directional intensity and anisotropy, as a function of time, at $r=1 \mathrm{AU}$. The solutions are shown at an angle of optimal magnetic connection and two points $\pm 45^{\circ}$ away from it, as indicated in the legend. Right: The calculated omni-directional intensity as a function of azimuthal angle at $t=0.5 \mathrm{hrs}$ for the different choices of $D_{\perp}$. The green Gaussian curve shows the injection function, while the vertical black line shows the azimuthal position of best magnetic connectivity to the source. Solutions are shown for different levels of perpendicular diffusion as determined by $\eta$.

\section{Introduction}

Recent observations by e.g. [1] and [2] have shown that, even for impulsive SEP events, the longitudinal spread of the particles are much larger that previously thought, even extending to almost $360^{\circ}$ in longitude at $1 \mathrm{AU}$. A possible process responsible for the observed strong longitudinal transport of these SEPs is effective diffusion perpendicular to the mean Parker heliospheric magnetic field. In this proceeding, we present model solutions of simulated impulsive SEP events in the ecliptic plane of the heliosphere, where perpendicular diffusion of SEPs in an undisturbed Parker HMF geometry is included.

\section{Modelling results}

We briefly summarize the modelling results of [4] and the reader is referred to this paper for more details. Fig. 1 (left panel) shows the assumed injection function (panel a), the calculated intensity (panel b) and anisotropy (panel c) as a function of time at $1 \mathrm{AU}$. Three solutions are shown, corresponding to different azimuthal positions: Optimal magnetic connection (solid black lines) and two points $\pm 45^{\circ}$ away from it (dashed red and dash-dotted blue lines respectively). The intensity plot illustrates the fact that the intensity is not symmetric about the point of best magnetic connection, with the flux enhanced towards the west (higher values of $\phi$ ), as compared to an equivalent point towards the East (a negative shift in $\phi$ ). Fig. 1 (right panel) shows the inten- 

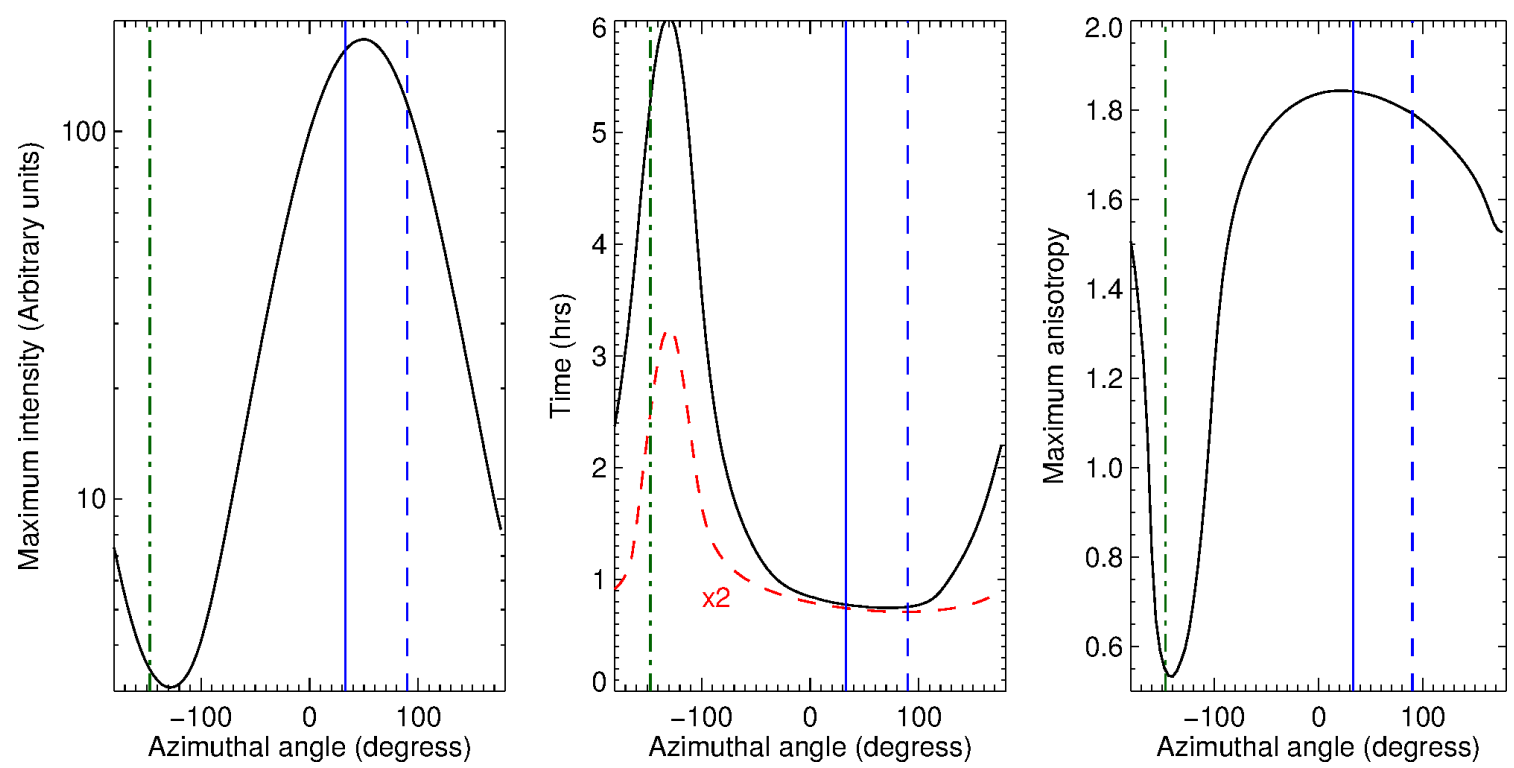

Figure 2: The figure shows, from left to right, the following quantities as a function of azimuthal angle at $r=1 \mathrm{AU}$ : The maximum intensity, both the time of maximum intensity and the onset time (note that the onset time is multiplied by a factor of 2) and the maximum anisotropy. The dashed blue line indicates where the injection function obtains its maximal value at the inner boundary, the solid blue line the position of optimal magnetic connectivity at $1 \mathrm{AU}$ and the dash-dotted line the position of worst magnetic connectivity.

sity as a function of $\phi$ (azimuthal angle) at $t=1 \mathrm{hr}$ and $r=1 \mathrm{AU}$. Three solutions are shown, corresponding to different assumptions of $\eta$ (defined by $\lambda_{\perp}=\eta \lambda_{\|}$; a larger value of $\eta$ thus corresponds to more effective perpendicular diffusion), as indicated in the legend. It is clear that the distributions are neither symmetrical about their maxima (again, enhanced towards the west), nor does the azimuthal position of the maximum flux at $1 \mathrm{AU}$ occur at the position of optimal magnetic connectivity. The latter quantity is also shifted towards the west, while this shift is larger for larger values of $\eta$. Assuming $\eta=0.1$, calculations of observable quantities are shown in Fig. 2, again as a function of azimuthal angle at $r=1 \mathrm{AU}$. The solid vertical line shows the position of optimal magnetic connectivity at $1 \mathrm{AU}$ to the source, the green dash-dotted line the angle of worst magnetic connection and the dashed black line the position where the injection function reaches a maximum at the inner boundary. In the left panel, the maximum intensity is shown for each $\phi$. The maximum of this distribution is shifted towards the west of best magnetic connection. The middle panel shows the time of maximum (solid curve) and the onset time (multiplied by a factor of 2; dashed red line). Both of these time scales are roughly anti-correlated with the maximum intensity; a higher maximum intensity usually corresponds to a shorter propagation time and hence, a shorter onset time and a shorter time needed to reach this maximum intensity value. The right panel shows the maximum anisotropy, generally occurring close to the onset time. The maximum anisotropy is again anti-correlated with the propagation time scales. It is believed that SEPs that take longer to reach e.g. $1 \mathrm{AU}$, must suffer more (pitch angle and perpendicular) diffusion, and hence, the distribution of these particles becomes increasingly isotropic. 


\section{Conclusions}

When perpendicular diffusion is included in a SEP propagation model, the resulting distribution is asymmetrical in terms of longitude, with the intensities enhanced towards the west of optimal magnetic connectivity to the acceleration region (i.e. the source). The results are in qualitative agreement with the observations presented by e.g. [6], [7], [9] and [5]. The maximum intensity and maximum anisotropy seems to be correlated, while both are anti-correlated to the time of maximum and the onset time. These seem to be in qualitative agreement with the results of [5]. The modelled azimuthal dependence of the maximum anisotropy seems, furthermore, to be consistent with the results of [9].

\section{Acknowledgments}

RDS is supported through the National Research Foundation (NRF) of South Africa Thuthuka Program (Grant no. 87998). Opinions expressed and conclusions arrived at are those of the authors and are not necessarily to be attributed to the NRF. RDS acknowledges support from the Fulbright Visiting Scholar Program. We also appreciate discussions at the team meeting 'Superdiffusive Transport in Space Plasmas and its Influence on Energetic Particle Acceleration and Propagation' supported by the International Space Science Institute (ISSI) in Bern, Switzerland.

\section{References}

[1] N. Dresing et al., The Large Longitudinal Spread of Solar Energetic Particles During the 17 January 2010 Solar Event, Sol. Phys. 2812812012.

[2] W. Dröge et al., Wide longitudinal distribution of interplanetary electrons following the 7 February 2010 solar event: Observations and transport modeling, JGR 11960742014.

[3] H.-Q. He \& W. Wan, The Statistical and Numerical Study of the Longitudinally Asymmetric Distribution of Solar Proton Events Affecting the Earth Environment of 1996-2011, In Proc. to the $33^{\text {rd }}$ ICRC 2013.

[4] R.D. Strauss \& H. Fichtner, On Aspects Pertaining to the Perpendicular Diffusion of Solar Energetic Particles, ApJ 801292015.

[5] I.G. Richardson et al., $>25 \mathrm{MeV}$ Proton Events Observed by the High Energy Telescopes on the STEREO A and B Spacecraft and/or at Earth During the First Seven Years of the STEREO Mission, Sol. Phys. 28930592014.

[6] Lario, D., et al. 2006, Radial and Longitudinal Dependence of Solar 4-13 MeV and 27-37 MeV Proton Peak Intensities and Fluences: Helios and IMP 8 Observations, ApJ 6531532006.

[7] Lario, D., et al. 2013, Longitudinal and Radial Dependence of Solar Energetic Particle Peak Intensities: STEREO, ACE, SOHO, GOES, and MESSENGER Observations, ApJ 767412003.

[8] G. Wibberenz, G. \& H.V. Cane, Multi-Spacecraft Observations of Solar Flare Particles in the Inner Heliosphere, ApJ $\mathbf{6 5 0} 11992006$.

[9] N. Dresing et al., Statistical survey of widely spread out solar electron events observed with STEREO and ACE with special attention to anisotropies, A\&A 567 A27 2014. 